\title{
Desensibilización rápida en pacientes con hipersensibilidad a aspirina sometidos a angioplastía coronaria. Reporte de cuatro casos
}

\author{
NICOLÁS VEAS P. a , GONZALO MARTÍNEZ ${ }^{\text {b }, ~ J O R G E ~ J A L I L ~ M ., ~}$ \\ ALEJANDRO MARTÍNEZ S., PABLO CASTRO G.
}

\section{Rapid aspirin desensitization in patients with a history of aspirin hypersensitivity requiring coronary angioplasty. Report of four cases}

Aspirin use is necessary after a coronary angioplasty. It should not be used in patients with a history of hypersensitivity. However, rapid desensitization protocols have been reported to allow its use in such patients. One of these protocols consists in the administration of progressive doses of aspirin, from 1 to $100 \mathrm{mg}$ in a period of 5.5 hours, in a controlled environment. We report four male patients aged 45, 49, 59 and 73 years with a history of aspirin hypersensitivity, who were subjected to a coronary angioplasty. In all, the rapid aspirin desensitization protocol was successfully applied, allowing the use of the drug after the intervention without problems.

(Rev Med Chile 2013; 141: 255-259).

Key words: Angioplasty, balloon, coronary; Aspirin; Desensitization, immunologic; Drug hypersensitivity.

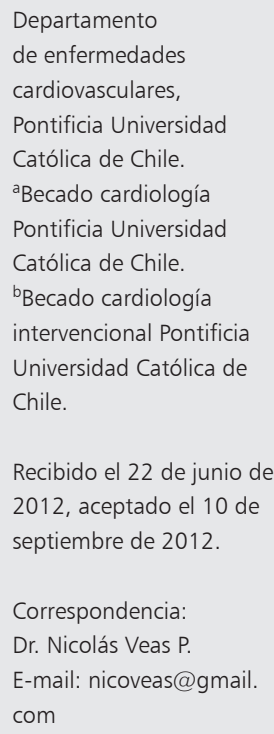

E 1 uso de aspirina es considerado un pilar fundamental en el tratamiento de la enfermedad coronaria ${ }^{1}$. Es especialmente necesaria cuando se realiza angioplastía coronaria. En este caso, en relación al traumatismo de la pared y al efecto de cuerpo extraño provocado por los stents, se produce activación plaquetaria y alto riesgo de trombosis, que se controla con el uso concomitante de aspirina y clopidrogel ${ }^{2}$. Un dilema terapéutico lo plantean los pacientes con alergia a la aspirina que tienen indicación de angioplastía coronaria. Afortunadamente, esta situación tiene una baja prevalencia, se estima entre 0,2 a $0,7 \%{ }^{3}$, pero cuando se presenta se dificultan las decisiones. Por un lado, la realización de la angioplastía sin su uso puede resultar en una complicación trombótica catastrófica ${ }^{4}$, y por otro, la reacción de hipersensibilidad a aspirina (HAAS), aunque puede tener una expresión benigna, como rinitis o rinosinusitis, asma o urticaria, también puede manifestarse como angioedema o anafilaxia ${ }^{3}$. Para estos pacientes se ha planteado una terapia de desensibilización previo a la intervención coronaria.

En este artículo comunicamos la experiencia de cuatro pacientes con antecedentes de HAAS y que se presentaron con un síndrome coronario agudo (SCA), siendo sometidos a un tratamiento de desensibilización rápida a aspirina (Tabla 1).

\section{Protocolo de desensibilización utilizado}

Existen diversos protocolos de desensibilización publicados en la literatura, algunos con mejores resultados que otros. En nuestro grupo se decidió utilizar el protocolo propuesto por Rossini y cols ${ }^{8}$, el cual detallaremos más adelante (Tabla 2). Es fundamental obtener las dosis descritas de aspirina, para ello se recomienda trabajar 
Tabla 1. Características de los pacientes sometidos a protocolo de desensibilización a aspirina

\begin{tabular}{|cccccccc|}
\hline $\begin{array}{c}\text { n de pa- } \\
\text { ciente }\end{array}$ & $\begin{array}{c}\text { Edad } \\
\text { (años)/ } \\
\text { género }\end{array}$ & $\begin{array}{c}\text { Síntomas } \\
\text { relacionados } \\
\text { a HAAS }\end{array}$ & $\begin{array}{c}\text { Presentación } \\
\text { de enfermedad } \\
\text { coronaria }\end{array}$ & $\begin{array}{c}\text { n de } \\
\text { arterias } \\
\text { coronarias } \\
\text { enfermas }\end{array}$ & $\begin{array}{c}\text { n de } \\
\text { stents }\end{array}$ & $\begin{array}{c}\text { Seguimiento } \\
\text { (meses) }\end{array}$ & $\begin{array}{c}\text { Dosis de } \\
\text { AAS en } \\
\text { último } \\
\text { seguimiento }\end{array}$ \\
\hline 1 & $59 / \mathrm{M}$ & Rash + AE & IAM s/ SDST & 1 & 1 & 14 & 325 \\
\hline 2 & $45 / \mathrm{M}$ & Rash gen & IAM S/SDST & 1 & 1 & 10 & 100 \\
\hline 3 & $49 / \mathrm{M}$ & Rash gen & IAM c/SDST & 1 & 1 & 3 & 100 \\
\hline 4 & $73 / \mathrm{M}$ & Angioedema & Infarto no Q & 2 & 2 & 4 & 100 \\
\hline
\end{tabular}

$N^{\circ}=$ número; $M=$ masculino; $F=$ femenino; HAAS = hipersensibilidad a aspirina; $A A S=$ Aspirina; gen = generalizado.

Tabla 2. Protocolo de desensibilización a aspirina ${ }^{8}$

\begin{tabular}{|cc|}
\hline Tiempo (min) & Dosis de aspirina $(\mathbf{m g})$ \\
0 & 1 \\
30 & 5 \\
60 & 10 \\
90 & 20 \\
210 & 40 \\
330 & 100 \\
\hline
\end{tabular}

en conjunto con el equipo quimicofarmacéutico de cada centro. Se reportan una baja incidencia de complicaciones, todas ellas leves. De las que se describen son exacerbaciones de cuadros asmáticos de base o reacciones cutáneas leves con buenas respuestas a tratamiento corticoidal o antihistamínico. Generalmente se presentan entre la tercera y cuarta dosis y si son reacciones severas como shock anafiláctico (no se describen en la literatura) se sugiere manejo habitual, finalizar el protocolo, monitorizar en Unidad Coronaria y buscar alternativas terapéuticas. Si la desensibilización se lleva a cabo, finalizando con las dosis crecientes, se debe reiniciar la aspirina en dosis habituales al día siguiente, tal y como se realiza en pacientes sin HAAS.

\section{Caso 1}

Varón de 59 años, con antecedentes de tabaquismo suspendido hacía 4 años y con historia de alergia a la aspirina caracterizado por eritema generalizado y edema glótico. La primera ocasión fue en el año 1974 y se vuelve a repetir con una dosis única el año 1983, motivo por el cual nunca más vuelve a recibir aspirina. Ingresa en noviembre de 2010 por angina inestable. Se realiza coronariografía que muestra estenosis significativa en arteria descendente anterior, ante lo cual se procede a realizar una angioplastía con stent en dicho vaso. Por antecedentes de alergia a la aspirina se deja con clopidogrel y cilostazol. Tres semanas posterior a su alta, presenta nuevo episodio de dolor torácico retroesternal opresivo prolongado y la coronariografía demuestra una trombosis intra-stent. Se trató con angioplastía con balón, bajo infusión de tirofibán endovenoso, consiguiendo un buen resultado clínico. Posterior a la intervención se realiza protocolo de desensibilización a aspirina, sin incidentes. Desde entonces se mantiene con aspirina y clopidrogel. Completado 1 año 2 meses de seguimiento, con buena adherencia a la terapia, no ha presentado efectos adversos, y se mantiene sin angina y sin evidencias de HAAS.

\section{Caso 2}

Varón de 45 años, fumador de larga data. En mayo del año 2011 ingresa por cuadro de dolor torácico opresivo retroesternal con síntomas neurovegetativos, de inicio súbito y moderada intensidad. Presenta ECG evolutivo, principalmente por infradesnivel en precordiales izquierdas, $y$ elevación enzimática. Ante el antecedente de alergia a la aspirina relatado por el paciente (eritema generalizado luego de dos dosis), se decide previo al estudio invasivo realizar protocolo de desensibilización, que se completa sin incidentes durante monitorización en Unidad Coronaria. Posteriormente, se realiza coronariografía que muestra lesión significativa en arteria circunfleja, que se trató con angioplastía e implante de stent. 
Evoluciona favorablemente sin complicaciones del procedimiento ni reacciones adversas a la aspirina mientras estuvo hospitalizado. Al cabo de 9 meses de seguimiento no ha presentado efectos adversos y se ha mantenido con buena adherencia a la terapia, sin angina y sin evidencias de HAAS.

\section{Caso 3}

Varón 49 años. Ingresa en noviembre de 2011 por cuadro de infarto agudo de miocardio (IAM) con SDST de pared inferior, Killip I de $3 \mathrm{~h}$ de evolución. Refiere antecedentes de alergia a la aspirina principalmente por eritema uriticarial generalizado que se ha presentado en 2 oportunidades. Se realiza coronariografía de urgencia que muestra lesión crítica en 1/3 medio de la arteria coronaria derecha, que se trató con angioplastía con stent sin incidentes. El mismo día se realiza protocolo de desensibilización a la aspirina. Posterior a la tercera dosis presentó prurito generalizado, que cede espontáneamente sin requerimientos de antihistamínicos. Su evolución posterior fue favorable y se dio el alta al quinto día post infarto. Luego de 3 meses de evolución presenta buena adherencia a la terapia, sin angina y sin evidencias de HAAS.

\section{Caso 4}

Varón de 73 años, portador de insuficiencia renal crónica en hemodiálisis desde hace 3 años. Cinco días previo a su consulta presenta cuadro de dolor torácico opresivo de $2 \mathrm{~h}$ de duración. El dolor se repite, con breve duración, durante sesión de diálisis. El ECG no mostró alteraciones significativas y la troponina I alcanzó a $23 \mathrm{ng} / \mathrm{ml}$ $(\mathrm{VN}<0,05 \mathrm{ng} / \mathrm{ml})$. Se hospitaliza en Unidad Coronaria con diagnóstico de SCA y se planifica estudio coronario invasivo. El paciente refiere haber utilizado aspirina 5 años antes, presentando rash alérgico generalizado luego de la primera dosis, que se acentúa luego de la dosis del día siguiente. Al tercer día presenta angioedema y edema glótico, requiriendo hospitalización para su manejo y suspensión de Aspirina. Se decide aplicar protocolo de desensibilización, sin reacciones adversas, y al día siguiente se realiza coronariografía que muestra estenosis severas en coronaria derecha distal y en tercio medio de descendente anterior. Se realizó angioplastia con implante de stents en ambas arterias. El paciente evoluciona sin complicaciones, recibiendo terapia antiplaquetaria dual bien tolerada. En el último control, 4 meses luego del evento, permanece en buenas condiciones con buena adherencia a la terapia, sin angina y sin evidencias de HAAS.

\section{Discusión}

Las reacciones de hipersensibilidad a aspirina están mediadas por inmunoglobulinas tipo $\operatorname{IgE}$ dirigidas a la droga o por producción excesiva de leucotrienos ${ }^{9}$. El segundo mecanismo se debe al bloqueo de la ciclooxigenasa-1 (COX-1) por aspirina. La COX-1 es necesaria para la síntesis de prostaglandina E2, la cual inhibe la 5-lipooxigenasa, una enzima responsable de la producción de leucotrienos que median la degranulación de mastocitos y la liberación de histamina y citoquinas. De esta manera, la inhibición de COX-1 por aspirina resulta en una actividad excesiva de 5-lipooxigenasa y la liberación de mediadores de los mastocitos, con el consiguiente riesgo de una reacción de hipersensibilidad ${ }^{5}$. La producción de IgE contra aspirina es un fenómeno menos entendido ${ }^{12}$.

La desensibilización a aspirina implica la exposición a dosis progresivamente mayores de esta droga, con el objetivo de reducir o eliminar la aparición de reacciones farmacológicas e inmunológicas. De esta manera, se produce una depleción controlada de mediadores inmunes y también el down-regulation de los receptores de leucotrienos ${ }^{6,7}$. Si bien existen distintos protocolos, nosotros utilizamos el propuesto por Rossini y cols ${ }^{8}$. Ellos lo evaluaron exitosamente en 26 pacientes con enfermedad coronaria que debían ser sometidos a angioplastía. A diferencia de otros métodos diseñados para pacientes electivos con reacciones respiratorias a aspirina ${ }^{9}$, el propuesto por Rossini y cols tiene la ventaja del escaso tiempo que tarda en completarse, lo cual puede ser crucial en pacientes con un SCA. En esta línea, Silberman $y$ cols $^{10}$ reportaron una serie de 16 pacientes desensibilizados a aspirina con un esquema abreviado que duraba entre 2,5 y $3,5 \mathrm{~h}$ y con una tasa de éxito superior a 90\%. Sin embargo, se presentaron dos casos de reacciones posteriores a las cuatro horas de la primera dosis, que requirieron uso de adrenalina y anti-histamínicos. El protocolo que utilizamos, que tarda $5,5 \mathrm{~h}$, nos parece razonable para pacientes que se encuentran cursando un SCA, ya que permite monitorizar adecuadamente 
la presencia de reacciones sub-agudas a la droga, empezar con una dosis muy segura y, a la vez, no retardar significativamente la realización del cateterismo coronario. Además, este protocolo no requiere el uso profiláctico de anti-histamínicos ni esteroides, pero debe ser realizado en condiciones vigiladas, idealmente en una unidad monitorizada y con equipamiento de reanimación adecuado en caso de requerirse. Evidentemente, un esquema de desensibilización de este tipo no debiera retardar la atención adecuada de los pacientes que se presentan con un infarto con supradesnivel del segmento ST, situación en la cual la intervención coronaria inmediata ha mostrado un enorme impacto pronóstico ${ }^{11}$. En estos pacientes, tal como ocurrió con el individuo del caso 3, se ha probado con éxito la desensibilización en los días posteriores al evento?.

Luego de completar exitosamente el esquema de desensibilización, se pueden ingerir dosis terapéuticas de aspirina indefinidamente. Sin embargo, debe ponerse atención en que el efecto de la tolerancia es temporal, existiendo el riesgo de re-sensibilización en caso de que la aspirina sea suspendida, aún cuando sea por un breve plazo de tiempo (2 a 7 días). De este modo, los pacientes deben ser advertidos de no suspender la droga y, en caso de hacerlo, que requerirán un nuevo ciclo de desensibilización ${ }^{7}$. Luego de la desensibilización debe iniciarse la aspirina en dosis habituales y al día siguiente tal y como lo realizarían en pacientes sin HAAS. Como dijimos anteriormente, la presencia de reacciones adversas en poco probable y de presentarse son leves. En nuestra experiencia tan sólo 1 paciente refirió prurito, el cual fue autolimitado, sin requerir terapia medicamentosa. Dado que este protocolo es reciente y aun poco aplicado en nuestro país, sugerimos realizarlo siempre monitorizado y en caso de presentar alguna reacción adversa no dudar en el uso de corticoides o antihistamínicos. De presentarse un shock anafiláctico, debe tratarse como tal, suspender el protocolo y buscar terapias alternativas a la aspirina, aunque esto no ha sido descrito ni en la literatura ni en nuestra serie. Es fundamental el tener las dosis descritas. Para esto nosotros lo realizamos con la cooperación de quimicofarmaceutas de nuestro hospital quienes lograron obtener las dosis necesarias sin mayores problemas en papelillos en las dosis descritas.

Este protocolo en la actualidad lo estamos aplicando a todo paciente que requiera cateterismo.
Evidentemente puede ser realizado a cualquier paciente con antecedentes de HAAS y que requiera uso de aspirina.

En nuestro conocimiento, esta es la primera experiencia comunicada en nuestro país sobre el uso de un protocolo de desensibilización en pacientes con HAAS. Creemos que este recurso es de gran utilidad para aquellos pacientes que requieren una angioplastia coronaria y tienen antecedentes claros de reacciones de hipersensibilidad con aspirina. Su correcta y cuidadosa aplicación puede permitir que obtengan los excelentes resultados actuales de la angioplastía coronaria y así evitar que un SCA se transforme en una catástrofe.

\section{Referencias}

1. Wijns W, Kolh P, Danchin N, Di Mario C, Falk V, Folliguet T, y cols. Guías de práctica clínica sobre revascularización miocárdica. Rev Esp Cardiol 2010; 63: 1485. e1-e76.

2. Mehta SR, Yusuf S, Peters RJ, Bertrand ME, Lewis BS, Natarajan MK, et al. Effects of pretreatment with clopidogrel and aspirin followed by long-term therapy in patients undergoing percutaneous coronary intervention: the PCI-CURE study. Lancet 2001; 358: 527-33.

3. Jenkins C, Costello J, Hodge L. Systematic review of prevalence of aspirin induced asthma and its implications for clinical practice. BMJ. 2004; 328: 434.

4. Holmes, D, Kereiakes DJ, Garg S, Serruys PW, Dehmer GJ, Ellis SG, et al. Stent Thrombosis. JACC 2010; 56: 1357-65.

5. Page NA, Schroeder WS. Rapid desensitization protocols for patients with cardiovascular disease and aspirin hypersensitivity in an era of dual antiplatelet therapy. Ann Pharmacother 2007; 41: 61-7.

6. Stevenson DD, Szczeklik A. Clinical and pathologic perspectives on aspirin sensitivity and asthma. J Allergy Clin Immunol 2006; 118: 773-86.

7. Wong JT, Nagy CS, Krinzman SJ, Maclean JA, Bloch KJ. Rapid oral challenge-desensitization for patients with aspirin-related urticaria-angioedema. J Allergy Clin Immunol 2000; 105: 997-1001.

8. Rossini R, Angiolillo DJ, Musumeci G, Scuri P, Invernizzi P, Bass TA, et al. Aspirin Desensitization in Patients Undergoing Percutaneous Coronary Interventions With Stent Implantation. Am J Cardiol 2008;101: 786-9.

9. Gollapudi RR, Teirstein PS, Stevenson DD, Simon RA. Aspirin sensitivity: implications for patients with coro- 
nary artery disease. JAMA 2004; 292: 3017-23.

10. Silberman S, Neukirch-Stoop C, Steg PG. Rapid Desensitization Procedure for Patients With Aspirin Hypersensitivity Undergoing Coronary Stenting. Am J Cardiol 2005; 95: 509-10.

11. Stone G. Angioplasty Strategies in ST-Segment_Eleva- tion Myocardial Infarction: Part I: Primary Percutaneous Coronary Intervention. Circulation 2008; 118; 538-51.

12. Szczeklik A, Stevenson DD. Aspirin-induced asthma: advances in pathogenesis, diagnosis and management. J Allergy Clin Immunol. 2003; 111: 913-21. 